\title{
A coronal explosion on the flare star CN Leonis
}

\author{
J. H. M. M. Schmitt ${ }^{1}$, F. Reale ${ }^{2}$, C. Liefke ${ }^{1}$, U. Wolter ${ }^{1}$, B. Fuhrmeister ${ }^{1}$, A. Reiners ${ }^{3}$, and G. Peres ${ }^{2}$ \\ 1 Hamburger Sternwarte, Gojenbergsweg 112, 21029 Hamburg, Germany \\ e-mail: jschmitt@hs.uni-hamburg.de \\ 2 Dipartimento di Scienze Fisiche e Astronomiche, Università di Palermo, Piazza del Parlamento 1, 90134 Palermo, Italy \\ 3 Inst. f. Astrophysik Universität Göttingen, 37077 Göttingen, Germany
}

Received 7 November 2007 / Accepted 24 January 2008

\section{ABSTRACT}

\begin{abstract}
We present simultaneous high-temporal and high-spectral resolution observations of the nearby flare star CN Leo at optical and soft X-ray wavelengths. During our observing campaign a major flare occurred, raising the star's instantaneous energy output by almost three orders of magnitude. The flare shows the often observed impulsive behavior, with a rapid rise and slow decay in the optical and a broad soft X-ray maximum about 200 seconds after the optical flare peak. In addition to this usually encountered flare phenomenology we find, however, an extremely short $\left(\tau_{\mathrm{dec}} \approx 2 \mathrm{~s}\right)$ soft X-ray peak, which is very likely of thermal, rather than nonthermal nature and coincides temporally with the optical flare peak. While at hard X-ray energies nonthermal bursts are routinely observed on the Sun at flare onset, thermal soft X-ray bursts on time scales of seconds have never been observed in a solar, nor stellar context. Timedependent, one-dimensional hydrodynamic modeling of this event requires an extremely short energy deposition time scale $\tau_{\text {dep }}$ of a few seconds to reconcile theory with observations, thus suggesting that we are witnessing the results of a coronal explosion on CN Leo. Thus the flare on CN Leo provides the opportunity to observationally study the physics of the long-sought "micro-flares" thought to be responsible for coronal heating.
\end{abstract}

Key words. X-rays: stars - stars: individual: CN Leo - stars: flares - stars: coronae - stars: activity

\section{Introduction}

The basic energy release in solar and stellar flares is thought to occur in the coronal regions of the underlying star through conversion of non-potential magnetic energy through magnetic reconnection (Priest \& Forbes 2002). Particle acceleration takes place and the accelerated particles and/or plasma waves move along the magnetic field lines and reach and heat cooler atmospheric layers (Syratovskii \& Shmeleva 1972; Brown 1973). Because of their small cooling times the heated photospheric layers start radiating immediately, serving as a proxy indicator for nonthermal particles, while the heated dense chromospheric layers "evaporate", leading to a soft X-ray flare (Brown 1973; Neupert 1978; Peres 2000). The heating and cooling time scales and the dynamic response of the various atmospheric layers are important for our understanding of solar and stellar flares and of coronal heating (Kostiuk \& Pikelner 1974; Somov et al. 1981; Tamres et al. 1986; Fisher 1987); a popular hypothesis (Klimchuk 2006; Parker 1988) even attributes all of the required energy input to the quiescent solar corona to (nano)-flare input.

Solar and stellar flares are observed to occur over vastly different time scales, ranging from a few seconds (Vilmer et al. 1994; Schmitt et al. 1993) to more than a week (Kürster \& Schmitt 1996), and the response of the heated plasma depends sensitively on the mode of energy input. Since the flare process involves plasma differing in temperature and density by more than four orders of magnitude, varying on short spatial and temporal scales, simultaneous multiwavelength data with sufficient spectral coverage and sufficient spectral and temporal resolution are required for any sensible observational diagnostics. Since the primary energy release and energy loss process of a flare is coronal, space-based X-ray observations are required. Using ESA's XMM-Newton X-ray observatory and ESO's Ultraviolet-Visual Echelle Spectrograph (UVES) on Kueyen on May 19, 2006, we observed a spectacular flare on the nearby $(d=2.39 \mathrm{pc})$ flare star CN Leo (spectral type: M5.5-M6.0, $T_{\text {eff }}=2800-2900 \mathrm{~K}$ ). Despite its low apparent rotational velocity of $v \sin i<3.0 \mathrm{~km} \mathrm{~s}^{-1}$ (Fuhrmeister et al. 2004), $\mathrm{CN}$ Leo shows all the attributes of a magnetically active star, including chromospheric and coronal emission as well as flaring in the optical and in the X-ray bands (Fuhrmeister et al. 2007).

\section{Observations and data reduction}

The XMM-Newton observatory carries three co-aligned X-ray telescopes and a co-aligned smaller optical telescope equipped with the Optical Monitor (OM); this satellite and its instruments are described in detail in a Special Issue of Astronomy \& Astrophysics Vol. 365 (Jan. 2001). For the CN Leo observations, we used the medium filter in Full Frame and Large Window mode for all EPIC observations. The OM was operated in fast window mode, allowing $U$ band flux measurements with a time resolution of $1 \mathrm{~s}$, limited by the chosen read-out sequence. We reduced the X-ray data with the XMM-Newton Science Analysis System (SAS) software, version 7.0. The basic XMM-Newton data consist of individual photons with known arrival time, position, and energy; using standard filtering criteria these data are further processed into EPIC lightcurves and spectra.

UVES is a cross-dispersed echelle spectrograph mounted on the Nasmyth B focus of the Kueyen telescope (UT2) covering the wavelength range from about $3000 \AA$ to $11000 \AA$ with a typical spectral resolution of $\sim 40000$. The temporal resolution of the high-resolution spectra is determined by the sum of exposure and read-out times (a few minutes in our case), a small 
fraction of the optical light is directed into an exposuremeter for each of the two spectral bands ("blue" and "red"), thus providing broadband photometry with a time resolution of one second. These data are, however, taken mostly for engineering purposes and are not corrected for background or airmass. For our analysis, we used the UVES pipeline products with the wavelength calibration carried out using thorium-argon spectra resulting in an accuracy of $\sim 0.03 \AA$ in the blue arm and $\sim 0.05 \AA$ in the red arm. Absolute flux calibration is based on the UVES master response curve and extinction files provided by ESO.

\section{Results}

\subsection{X-ray and optical light curves}

In Fig. 1 we plot - from bottom to top - the recorded XMM-Newton X-ray light curve (i.e., the sum of the $\mathrm{PN}+\mathrm{MOS} 1+\mathrm{MOS} 2$ detectors), the XMM-Newton Optical Monitor (OM) $U$ band light curve, and the UVES blue and red light curves for part of our observing run. In each panel we show a linear light curve covering $30 \mathrm{~s}$ around the flare onset, while the respective inserts show the logarithmic light curves of the whole flare event including some quiescence. The most notable feature in these light curves is the enormous, simultaneously occurring intensity increase in all considered energy bands starting at around UT $=23: 46: 20$. The relative increase in terms of flare peak to pre-flare flux is $>100$ in X-rays and $\approx 750$ in the UVES "blue" band. Even in flare stars, flares with magnitude increases $>7$ as shown in Fig. 1 are quite rare, and we are actually not aware of any examples in the literature sampled with the time resolution and spectral coverage of our data. The temporal alignment of the light curves in different bands ought to be accurate to at least $0.5 \mathrm{~s}$ and hence below the bin width. Figure 1 shows four remarkable properties of our multiwavelength light curves: first, the flare onset appears to be earlier in the optical wavebands, in particular, there is no evidence for any significant soft X-ray "precursor" activity, while there is some "pre-flash" visible in the $U$ band; second, the rise from quiescence to flare peak in the optical is 750 fold and occurs within $\approx 10 \mathrm{~s}$; third, both the "red" and "blue" UVES light curves show two peaks separated by $5 \mathrm{~s}$; and fourth, the first optical peak is accompanied by a soft X-ray peak. Note that this first soft X-ray peak is very sharp, lasting at most $\sim 2 \mathrm{~s}$, and does not coincide with the main soft X-ray flare peak, which in fact is quite flat and occurs about $200 \mathrm{~s}$ later (cf., insert in bottom panel of Fig. 1).

\subsection{The spectral nature of the $X$-ray burst}

The crucial question concerning the X-ray light curve is the nature of the soft X-ray peak at time $T=15 \mathrm{~s}$ in Fig. 1 . We emphasize that this peak is statistically highly significant. We find a total of 105 counts in the two adjacent $1 \mathrm{~s}$ bins around the optical flare maximum. A smooth interpolation of the flare rise light curve leads us to a "background" estimate of $\approx 55$ counts in those two bins, implying an extremely high statistical significance of the $2 \mathrm{~s}$ flare peak. Accepting the X-ray and optical peak and their close temporal association as real, on the one hand would suggest a nonthermal origin of the observed X-ray emission, while on the other hand, nonthermal X-ray emission has never been conclusively demonstrated from stars (Osten et al. 2007). Also, impulsive soft X-ray radiation from the Sun is known only on much longer time scales (Hudson et al. 1994). On the one hand, nonthermal, often impulsive X-ray emission is routinely observed from solar flares, with the the discrimination between thermal and nonthermal emission becoming exceedingly difficult (Kahler 1975) below $\sim 25 \mathrm{keV}$. On the other hand, assuming a thermal origin of the first X-ray peak, makes the observed fast decay time of $\sim 2 \mathrm{~s}$ difficult to reconcile with the usually invoked conductive or radiative cooling time scales.

Unfortunately, the spectral analysis of the EPIC pulse height data in the respective time interval from UT 23:46:36 to UT 23:46:44 is plagued by the low SNR in this short stretch of data. The rebinned EPIC-pn spectrum of this time interval is shown in Fig. 2, together with a best fit assuming either a thermal or a power-law spectral shape. For the thermal fit we assumed a two-temperature model with solar abundance, incidentally, a one-temperature component model with variable abundance also yields fits of comparable statistical quality. The fit quality of a power-law fit $\left(\chi_{\text {red }}^{2}=2.0\right)$ is clearly far inferior to that of the thermal models $\left(\chi_{\text {red }}^{2}=0.9\right)$, and an improvement in quality of a power-law fit can only be brought about by the introduction of substantial amounts of cold absorption $\left(N_{\mathrm{H}} \sim 4 \times 10^{21} \mathrm{~cm}^{-2}\right)$, which we regard as utterly unphysical in the context of $\mathrm{CN}$ Leo. Therefore, we clearly prefer a thermal interpretation of the "burst" spectrum purely on observational grounds. This thermal interpretation is supported by the X-ray spectrum between UT 23:46:44 to UT 23:47:44, immediately after the burst, displayed in Fig. 3, together with the power fit producing an acceptable fit to the burst spectrum in Fig. 2. Clearly, this spectrum cannot possibly be fit with a power-law spectrum, the iron L complex and $\mathrm{Ne}$ lines in the energy range $0.7-1.2 \mathrm{keV}$ prevent a power-law description; formally, a powerlaw fit with variable slope results in $\left(\chi_{\text {red }}^{2}=3.32\right)$, while a thermal spectrum yields $\left(\chi_{\text {red }}^{2}=1.01\right)$ and thus we conclude that the spectrum between UT 23:46:44 to UT 23:47:44 is definitely thermal. Going back to Fig. 2, we realize that the largest powerlaw fit residuals are found near $1 \mathrm{keV}$, precisely in that spectral range where the thermal nature of the post-burst spectrum becomes most apparent. If we assumed the burst spectrum to be nonthermal, the best fit power-law slope of $\gamma=2$ of the burst spectrum (cf., Fig. 2) would have to agree only coincidentally with the "slope" of the thermal spectrum in the post burst spectrum (cf., Fig. 3). Therefore, we conclude that the EPIC pulse height spectra provide no evidence for a nonthermal character of the observed X-ray emission at any time during the flare.

\subsection{Optical burst spectrum}

We obtained a high-resolution UVES spectrum, covering the time interval UT 23:30:35 to UT 23:47:15 and the wavelength range between $3200 \AA-3850 \AA$ (shown in Fig. 4). While this spectrum covers also periods of quiescence, it is clearly dominated by the flare and can be taken as a representative mean spectrum of the first minute of flare emission. Over this wavelength range, the emission is almost linearly decreasing with wavelength, without any major emission lines except the Balmer lines at the long wavelength end; the bulk of the chromospheric emission lines appears only in later spectra. The slope of the best fit blackbody spectrum corresponds to an effective temperature of $\sim 11320 \mathrm{~K}$; other fits with temperatures fixed at $10000 \mathrm{~K}$ and $15000 \mathrm{~K}$ are also shown. Converting then the observed spectral normalization to an emission area (using the known distance of CN Leo) results in an area estimate of $\approx 4.4 \times 10^{18} \mathrm{~cm}^{2}$, while the other temperatures lead to values between $1.7-7.1 \times 10^{18} \mathrm{~cm}^{2}$. Some caution is warranted in this estimate: As is evident from Fig. 1, significant photospheric temperature changes, that cannot be resolved in the UVES spectrum, take place during the 


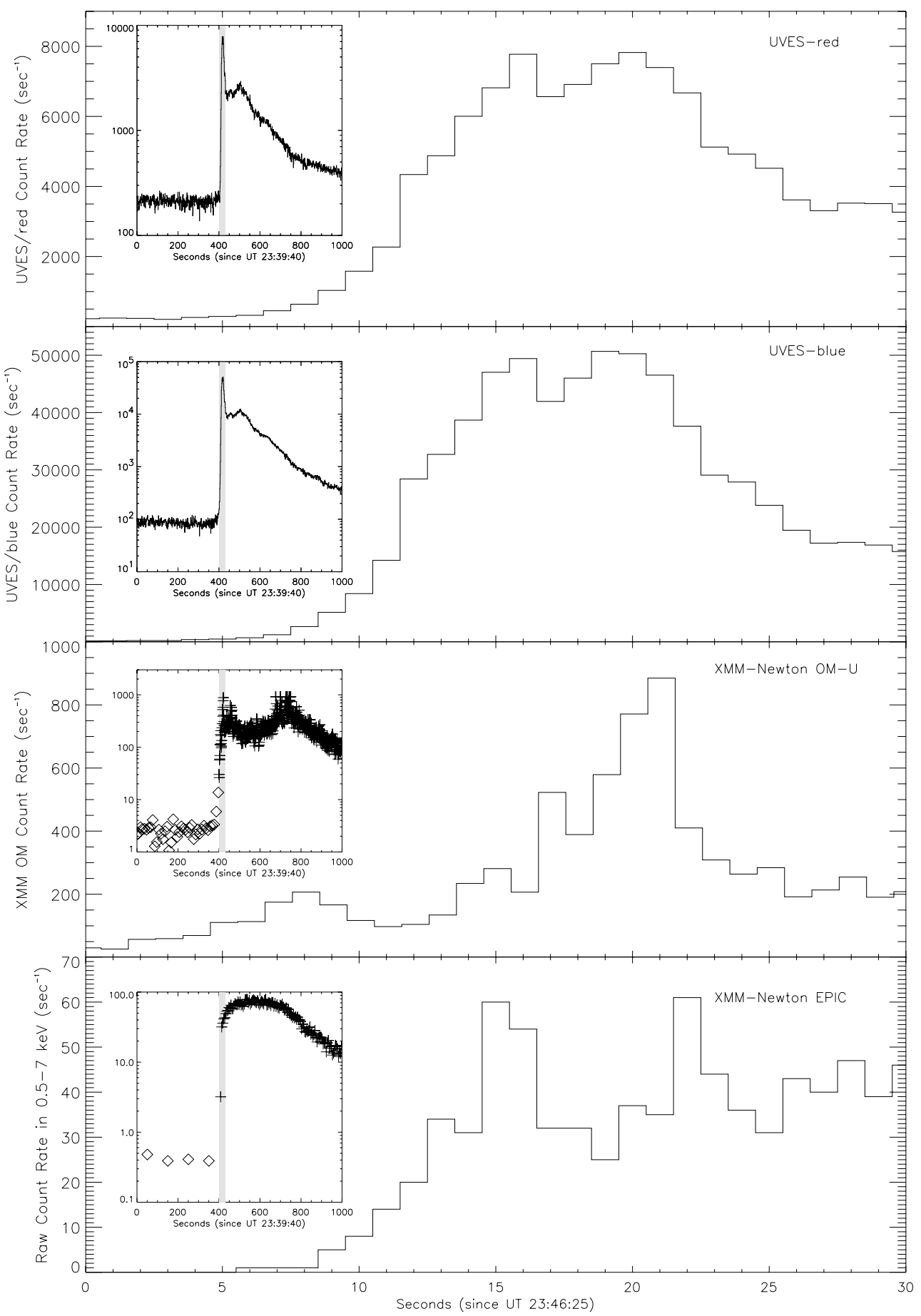

Fig. 1. XMM-Newton EPIC raw X-ray (sum of PN, MOS1, MOS2 detectors) and OM $U$ band light curves (bottom panels), UVES blue and red channel (upper panels) during the first $30 \mathrm{~s}$ of the impulsive flare on CN Leo; all times are in UT seconds relative to May 19, 2006, 23:46:25; the inserts show the same light curves covering $1000 \mathrm{~s}$ relative to May 19, 2006, 23:39:40 in logarithmic scale, including some quiescent emission and the full flare light curve; the light curve portions displayed enlarged are gray shaded. The X-ray light curves exclude events recorded in the center of the point response function to avoid pile-up effects; the total X-ray count rate is almost twice as large. The binning of all data is $1 \mathrm{~s}$, except that of the X-ray data in the insert, which is set to $100 \mathrm{~s}$ prior to the flare onset and to $10 \mathrm{~s}$ afterwards. The OM light curve is dead time corrected; for rates above $500 \mathrm{cts} / \mathrm{s}$ these corrections and the lightcurve become unreliable.

first $60 \mathrm{~s}$. The derived temperature is, therefore, a mean and probably lower than the achieved maximum temperature. Further, no calibration spectra of standard stars were taken during the same night and using the spectra of different standard stars taken during different nights leads to errors of up to $50 \%$ in the absolute calibration. We, therefore, conclude that the emission area responsible for the observed blue optical emission was in the range $1-10 \times 10^{18} \mathrm{~cm}^{2}$. An independent estimate of the size of this emission area can be derived from the UVES photometer data. Using the mean temperature derived from the spectrum and the count rate during the spectral exposure, we can convert the observed count rate into an energy flux (from a comparison with other stars) and derive in this fashion an emission area of $\approx 8 \times 10^{18} \mathrm{~cm}^{2}$, in good agreement with the estimate 


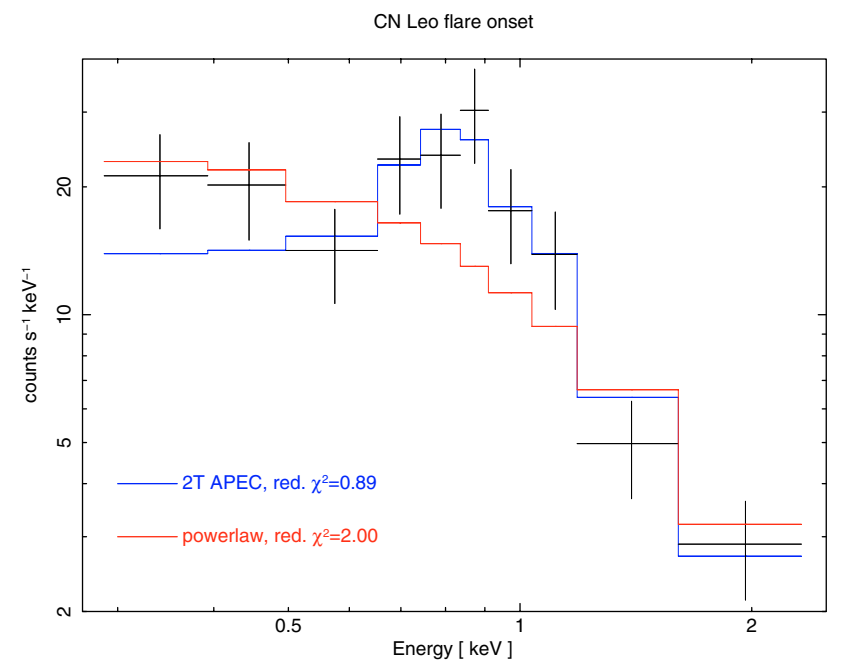

Fig. 2. EPIC pn spectrum of burst between UT 23:46:36 to UT 23:46:44 (black data points) together with model fit assuming a thermal spectrum (blue curve) and a power-law (red curve).

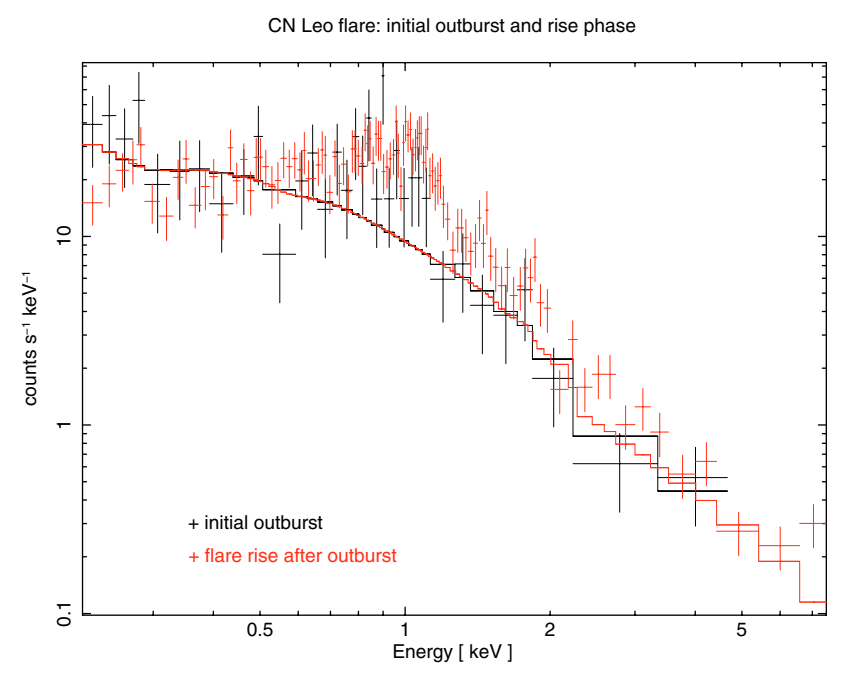

Fig. 3. Comparison of burst spectrum between UT 23:46:36 to UT 23:46:44 (black data points) with postburst spectrum (red data points) between UT 23:46:44 to UT 23:47:44; a power-law fit with photon index $\gamma=2$ is also shown.

from the spectrum. We thus conclude that the photospheric flare peak temperature was in excess of $11000 \mathrm{~K}$ and covered a flare area $A_{\mathrm{fl}}$ of $1-10 \times 10^{18} \mathrm{~cm}^{2}$, and obviously one expects the X-ray emission to arise from a similarly-sized region. Using the pile-up corrected PN light curve we can use the observed flare count rate in the energy band $0.5-8 \mathrm{keV}$ to compute an (isotropic) radiative loss of $\approx 3.8 \times 10^{31} \mathrm{erg}$; we estimate that about two thirds of the overall radiative losses are contained in this energy band. During the first X-ray peak, the recorded X-ray luminosity is estimated to be $\sim 5 \times 10^{28} \mathrm{erg} \mathrm{s}^{-1}$ (Fig. 6).

\section{Modeling and interpretation}

\subsection{Model ansatz}

To address the question whether our observations and the above derived physical parameters are consistent with the expected hydrodynamic response of coronal plasma to a fast transient heat flux, we performed a hydrodynamical simulation of an initially hydrostatic loop with prescribed length $L=4 \times 10^{9} \mathrm{~cm}$ and apex

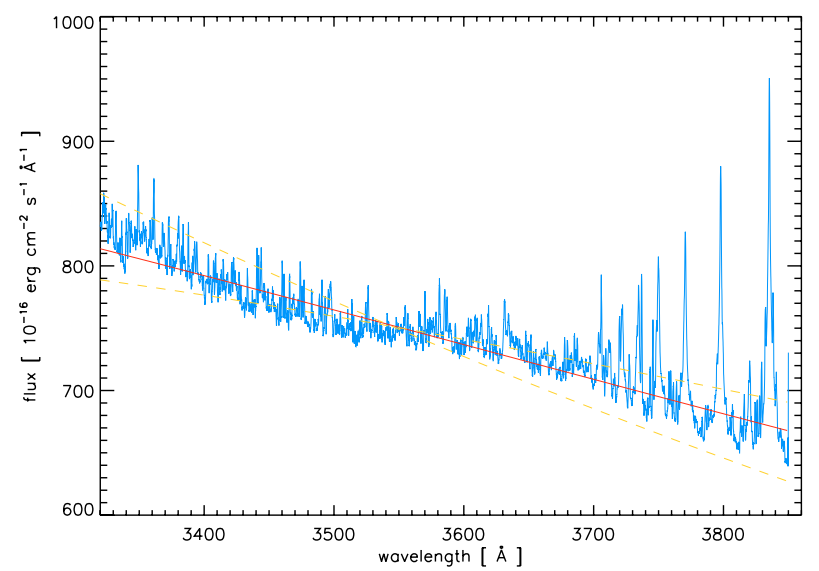

Fig. 4. VLT/UVES spectrum (between UT 23:30:35 to 23:47:15) covering the wavelength range between $3300-3850 \AA$. Data points are shown as blue histogram, black body fits with temperatures of $11318 \mathrm{~K}$ in red and for $10000 \mathrm{~K}$ and $15000 \mathrm{~K}$ as dashed lines. Balmer line emission is visible at longer wavelengths, but at shorter wavelengths most of the flux resides in continuum.

temperature $T_{\max }=2 \times 10^{6} \mathrm{~K}$ subjected to impulsive heating. For our hydrodynamical simulations we use the Palermo-Harvard (PH) code (Peres et al. 1982; Betta et al. 1997), which solves the time-dependent 1D hydrodynamic equations on an Eulerian grid. The energy equation includes the effects of optically thin radiative losses, heating and thermal conduction, treated in the Spitzer form, which should apply in a high-density medium, where the heat pulse is deposited, The heating term $Q$ consists of two contributions, a spatially and temporally constant term to produce a quiescent corona, and a spatially and temporally varying term to describe a flare. Since the microphysics of the heating is not well understood, we use an ad hoc parameterization of the form $Q_{\mathrm{fl}}=H_{0} f(t) g(s)$. For $g(s)$ we use a Gaussian $g(s)=\frac{1}{\sqrt{(2 \pi \sigma)^{2}}} \exp \left(\frac{-\left(s-s_{0}\right)^{2}}{2 \sigma^{2}}\right)$, centered at $s_{0}=2 \times 10^{8} \mathrm{~cm}$ (as measured from the loop footpoint) and $\sigma=10^{8} \mathrm{~cm}$, for $f(t)$ we use a symmetric triangular profile such that $f(t)$ differs from 0 for a time interval $t_{\text {heat }}$ and assumes a maximal value of unity and $H_{0}=7200 \mathrm{erg} \mathrm{cm}^{-3} \mathrm{~s}^{-1}$. The main difficulty for a numerical solution of the corona problem is the treatment of the transition region, where temperature and density gradients are extremely large. These numerical difficulties are aggravated by the fact that during the flare evolution the position of the transition region changes its spatial location. The PH code uses a re-adaptive grid, which insures a maximal relative change of $10 \%$ in all physical variables on adjacent grid points, provides a smoothly varying grid size over the whole computational domain, and samples the whole region of interest with typically 500-550 grid points. At the footpoints the model loop is anchored to a dense chromosphere, the structure of which follows the treatment by (Vernazza et al. 1981) and in which the energy balance is maintained throughout the simulation. We assume the loop to be symmetric with respect to its apex, so that the evolution is computed along one loop half and symmetry boundary conditions are imposed at the apex. The lower boundary is maintained at a fixed temperature at the chromospheric temperature minimum.

\subsection{Explosion modeling}

We specifically consider a simulation with $\tau_{\text {dep }}=10 \mathrm{~s}$ and a total deposited heat fluence $E_{\mathrm{tot}}=1.8 \times 10^{13} \mathrm{erg} / \mathrm{cm}^{2}$, assumed to be 


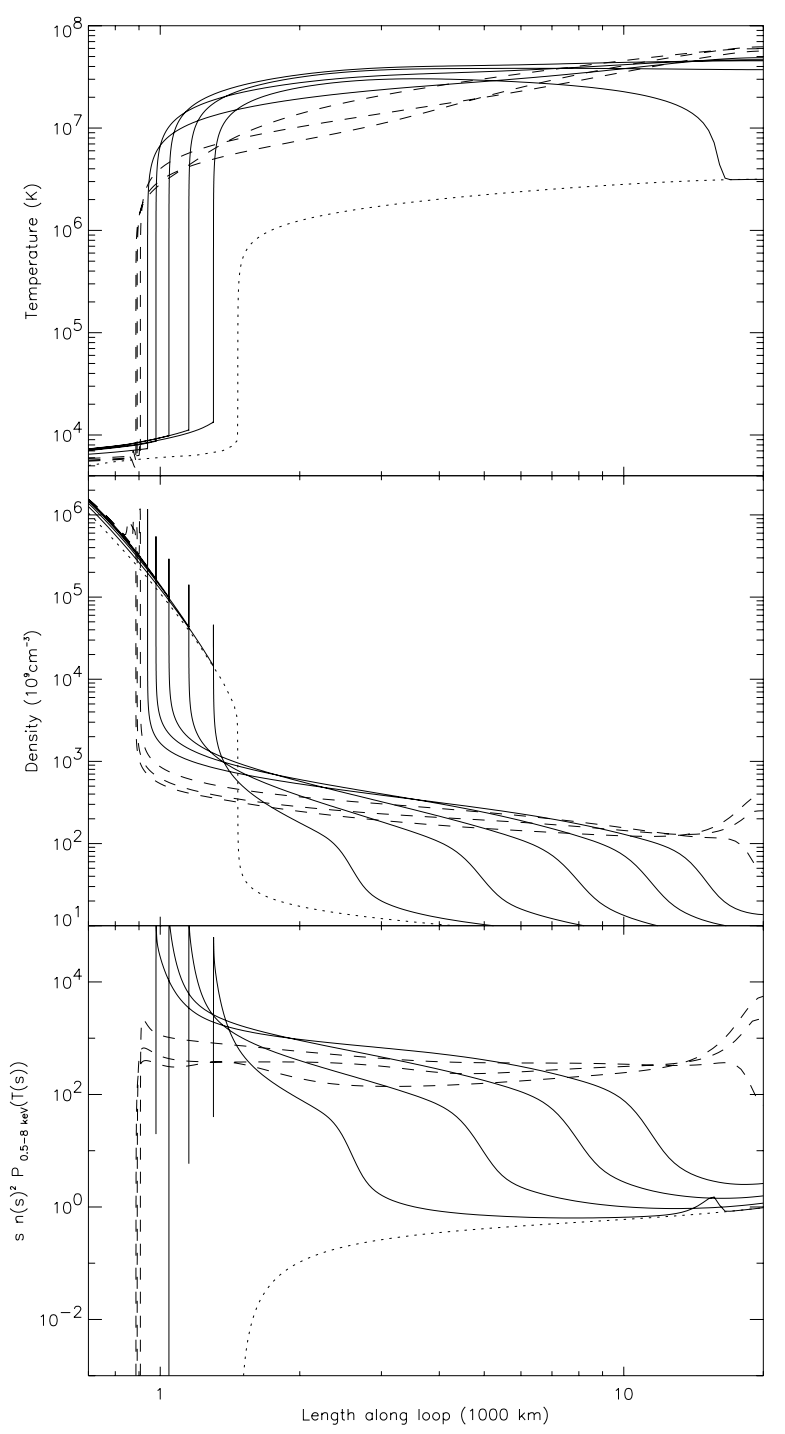

Fig. 5. Calculated run of temperature (upper panel), density (in units of $10^{9} \mathrm{~cm}^{-3}$; medium panel), and emission measure weighted cooling function (bottom panel) for the first $20 \mathrm{~s}$ of a coronal explosion. The initial state (a quiescent loop with $T_{\max }=3 \times 10^{6} \mathrm{~K}$ ) is shown as a dotted line, the evolution at times $t=2 \mathrm{~s}, 4 \mathrm{~s}, 8 \mathrm{~s}$, and $10 \mathrm{~s}$, during which heating takes place, is shown as solid lines, that at times $t=13 \mathrm{~s}$, $16 \mathrm{~s}$, and $19 \mathrm{~s}$ as long-dashed lines.

deposited at a height of $2000 \mathrm{~km}$ above the photosphere. The results of our simulation are shown in Fig. 5, where we plot the run of density and temperature vs. loop length for the initial state and every $2 \mathrm{~s}$ into the flare evolution. The main effect of the transient heating is a rapid temperature increase of the "old" (i.e., preflare) corona and transition region of the loop and parts of the "old" chromosphere to temperatures of 20-40 MK. Because of the high-density of the latter, a high-pressure region, sandwiched between the low-pressure "old" corona and low-temperature unheated chromosphere/photosphere, is formed at the bottom of the "old" transition region. An expansion wave starts propagating upwards with speeds in excess of $1000 \mathrm{~km} \mathrm{~s}^{-1}$ to increase the density of the "old" corona ("loop filling"). This explosive filling of the loop occurs on a time scale of $10 \mathrm{~s}$, comparable to the sound crossing time of the structure. Once the loop is rapidly filled, the material is more gently accumulated and compressionally heated, leading to a slower rise in emission measure and temperature. At the same time, the high-pressure "new"

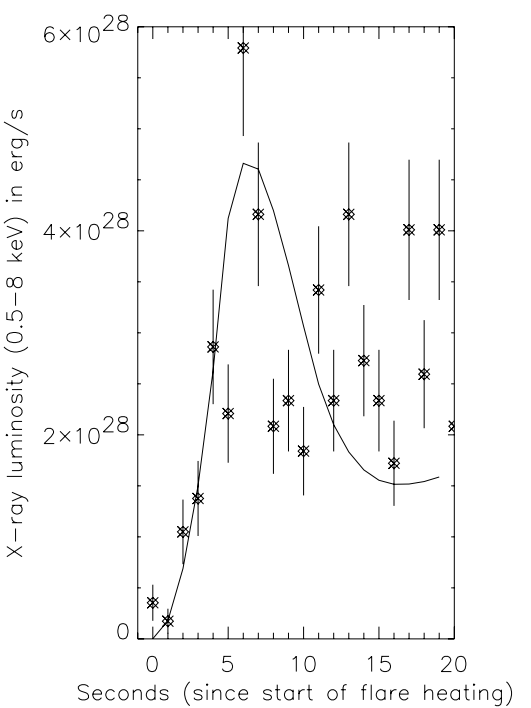

Fig. 6. XMM-Newton X-ray light curve (in erg/s) overplotted over hydrodynamic model prediction; time is with respect to the modeled start of the energy input. Note the good agreement between data and model although no best fit was performed. Also note that the event at $t=13 \mathrm{~s}$ might represent a further soft X-ray burst.

transition region moves inward into the chromosphere with speeds of $\sim 30 \mathrm{~km} \mathrm{~s}^{-1}$; a narrow density spike separates inward moving outer regions from not yet accelerated inner regions. Once the heating stops, the temperature in the lower loop portions drops through conductive losses into the chromosphere, while the temperature at the loop top still increases through compressional heating. From the runs of temperature and density we compute the temperature-dependent emission measure and cooling in the energy range $0.5-8 \mathrm{keV}$, displayed in Fig. 5. Obviously, during the first $\approx 10 \mathrm{~s}$, the main contribution to the $\mathrm{X}$-ray flux comes from the bottom parts of the loop, while at later times the evaporated plasma dominates the overall coronal emission.

\subsection{Comparison to observations and interpretation}

The theoretical curves displayed in Fig. 5 can be readily compared to our XMM-Newton data. It is straightforward to compute the total X-ray flux assuming some loop cross-sectional area and in Fig. 6 we juxtapose our model predictions (solid line) computed under the assumption of an emission area of $A_{\mathrm{fl}}=10^{19} \mathrm{~cm}^{2}$ and the observed X-ray luminosities computed from the pile-up corrected PN lightcurve using a count-rate-toflux conversion of $1.8 \times 10^{-12} \mathrm{erg} / \mathrm{cm}^{2} /$ count and the known distance towards CN Leo. Although no best fit (in terms of the temporal model evolution) has been attempted we note the good agreement between theoretical expectations and observations. The X-ray peak coincides with the end of the heating phase and the spike in the light curve at $t=13 \mathrm{~s}$ may represent another soft X-ray burst.

\subsection{Comparison to observations and interpretation}

The above interpretation of the XMM-Newton light curve is supported by the observed run of the evolution of count rate and mean photon energy for the first $100 \mathrm{~s}$ into the flare shown in Fig. 7. As is clear, the mean photon energy and, therefore, the mean X-ray temperature are decreasing, implying that the 


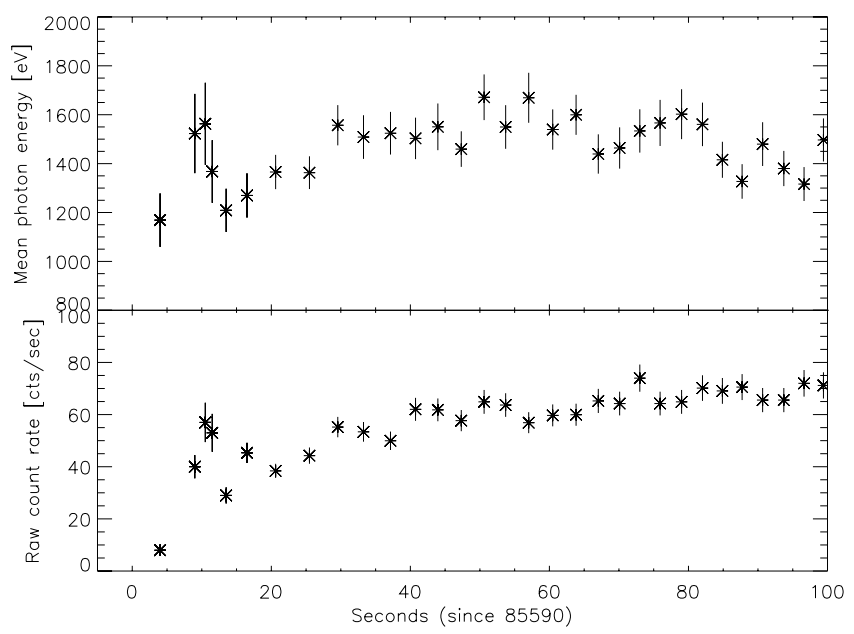

Fig. 7. XMM-Newton EPIC light curve (bottom panel) and mean photon energy (top panel) vs. time for the first $100 \mathrm{~s}$ around flare onset; all times are relative to May, 19th, 2006, 23:46:20. The mean photon energy has been derived from all photons with registered energies $>500 \mathrm{eV}$; each data point represents the mean energy of 200 photons.

X-ray emitting plasma is cooling. In the downward direction, conductive cooling is the dominant cooling process; computing the cooling time scale at the heat pulse center through conduction via $\tau_{\text {cond }}=\frac{21 n k}{2 \kappa_{0}} \frac{L^{2}}{T^{5 / 2}}$ we find $\tau_{\text {cond }} \approx 1.7 \mathrm{~s} \mathrm{using}$ $n=7 \times 10^{12} \mathrm{~cm}^{-3}, L=1 \times 10^{8} \mathrm{~cm}$ and $T=3.4 \times 10^{7} \mathrm{~K}$. The rapid cooling in the lower section of the loop leads to a pressure deficit accompanied by a density decrease and ensuing decrease in X-ray emission (cf., Fig. 5), driven by the fast evaporation front moving ahead. The short lasting soft X-ray burst shown in Fig. 1 is thus explained with a highly coherent plasma evolution, i.e., by flaring plasma confined in a single magnetic flux tube and by assuming that a major fraction or all of the observed energy release of $>3.8 \times 10^{31}$ erg occurred on a rather short time scale of $10 \mathrm{~s}$ or less. We, therefore, conclude that we are directly witnessing the process of evaporation as a result of the explosive heating of the lower chromosphere.

\subsection{Thick target bremsstrahlung}

As is clear from the above description, the energy input in our modeling occurs in an ad hoc fashion by specifying an arbitrary spatially and temporally varying heating function. Since the precise nature of the heating physics is unknown, this approach seems justified. Of course, the physical picture we have in the back of our minds is that of energetic particles (electrons) penetrating deep into the chromosphere and upper photosphere, dissipating their energy there and heating up the adjacent atmosphere. Since the UVES light curves are well co-aligned with the initial X-ray light curve and since the former emission comes from relatively cool material far extending into the red wavelength band, we require fairly energetic electrons to energize these photospheric layers. Unfortunately, the precise properties of these electrons and, in particular, their energy spectra and spectral cutoffs are only poorly known for solar flares and essentially unknown in the case of stellar flares. At any rate, one expects thick-target bremsstrahlung from this nonthermal particle population, but at what level? To this end we follow the calculations by Syratovskii \& Shmeleva (1972) and Brown (1973), who considered the bremsstrahlung X-ray spectra for bremsstrahlung emitted by a population of electrons injected into the flare region with an energy distribution function of the form

$F(E)=F_{0}\left(\frac{E_{0}}{E}\right)^{\gamma}$.

The approach taken by Brown (1973) is analytical; the most recent calculations of thick-target bremsstrahlung are those by Holman (2002), who computed microwave and X-ray spectra for such power-law electron distributions using the appropriate relativistic cross sections and considering the effects of low and high energy cutoffs. As demonstrated by Brown (1973) and Syratovskii \& Shmeleva (1972), an electron power-law distribution of the form given in Eq. (1) leads to a power-law distribution of the observed photon flux density $I(\epsilon)$ of the form

$I(\epsilon)=I_{0}\left(\frac{E_{0}}{\epsilon}\right)^{\beta}$,

i.e., again a power-law distribution. The respective power-law indices and normalizations are related through

$\beta=\gamma-1 \quad F_{0} E_{0}=\frac{\beta(\beta-1) I_{0}}{A B(\beta-1,1 / 2)}$

$B(\beta-1,1 / 2)$ in Eq. (3) denotes the Beta-function, and the constant $A$ is given by

$A=\frac{8}{3} \frac{r_{\mathrm{o}}^{2}}{137} \frac{m c^{2}}{\pi e^{4} Z^{2} \Lambda}$

where the quantities $c, r_{\mathrm{o}}, m, e, Z$, and $\Lambda$ denote speed of light, classical electron radius, electron mass, electron charge, ion charge number, and Coulomb logarithm respectively. Finally, the total energy content $E_{\text {tot }}$ of the electron population in the energy range from $E_{0}$ to infinity can be computed from

$E_{\mathrm{tot}}=\frac{I_{0} \beta E_{0}}{A B(\beta-1,1 / 2)}$

Thus, as well known from solar physics, the power-law index of the electron distribution as well as the normalization of the electron distribution and, therefore, its total energy content can be inferred from the observed photon power-law index $\beta$ and the observed photon fluence $I_{0}$, if the photons are indeed generated by the thick target bremsstrahlung mechanism. If we assume - for argument's sake - a thick target bremsstrahlung origin for the observed burst, we use the best-fit photon spectral index of $\beta \sim 2$ and the burst integrated photon fluence at $1 \mathrm{keV}$ of $4.8 \times 10^{37}$ photons $/ \mathrm{keV}$ to compute the total energy content $E_{\mathrm{pl}}$ of the power-law electron population of $2.4 \times 10^{35} \mathrm{erg}$ with a lowenergy cutoff of $1 \mathrm{keV}$; assuming a low-energy cutoff of $0.1 \mathrm{keV}$, we instead find $E_{\mathrm{pl}}=2.4 \times 10^{36} \mathrm{erg}$. Regardless of the assumed low-energy cutoff, the thus derived total energies are a few orders of magnitude above the observed soft X-ray energy output of $3.8 \times 10^{31} \mathrm{erg}$. In other words, under the assumption of nonthermal bremsstrahlung, we would have to assume that, unlike the solar case, the bulk of the energy contained in the nonthermal electron population goes into other (unobservable) forms of energy. Applying Occam's razor we thus conclude that both observations and theory suggest that the observed soft X-ray emission is not due to thick-target bremsstrahlung. At the same time we have shown that thick-target bremsstrahlung, which is expected to be produced at some level, can be easily accommodated within the existing observational framework, even if its low-energy cutoff were to extend down to below $1 \mathrm{keV}$ and, therefore, our theoretical modeling is fully consistent with the existing data. 


\subsection{Physical consistency of modeling}

Does our theoretical modeling capture all of the important physics of the flare explosion? A basic assumption in our modeling is the 1D character of the problem. Enormous pressure differences exist between "bottom" and "top" of the flaring plasma, while the magnetic field imposes a strong 1-dimensional directionality of the plasma along the field lines. Inspecting the maximally occurring gas pressures, we find values of up to $10000 \mathrm{dyn} \mathrm{cm}^{-2}$, requiring a magnetic field of $>300 \mathrm{G}$ for confinement. The mean photospheric field in CN Leo was simultaneously measured to exceed $2000 \mathrm{G}$ (Reiners et al. 2007), thus plasma confinement is no problem and our 1D modeling should be correct. The released energy is assumed to come from magnetic reconnection. An efficient energy conversion requires the incoming flow velocity to be less than $10 \%$ of the Alfven speed $v_{\mathrm{A}}$, and hence the time scale $\tau_{\text {diss }}$ for an efficient magnetic field dissipation on a spatial scale $L$ becomes $\tau_{\text {diss }} \sim \frac{L}{0.1 v_{\mathrm{A}}}$. Using $L \sim 2 \times 10^{9} \mathrm{~cm}$ and $\tau_{\text {diss }} \sim 5 \mathrm{~s}$, we find $v_{\mathrm{A}} \sim 4 \times 10^{9} \mathrm{~cm} / \mathrm{s}$, which appears unrealistically large. However, since we expect coronal fields on the order of $\approx 300 \mathrm{G}$ and particle densities of $\approx 5 \times 10^{8} \mathrm{~cm}^{-3}$, we find Alfven velocities of $v_{\mathrm{A}} \sim 3 \times 10^{9} \mathrm{~cm} / \mathrm{s}$, and therefore the assumption of Alfven speeds in excess to $10^{9} \mathrm{~cm} / \mathrm{s}$ is reasonable.

\section{Discussion and conclusions}

Heating by so-called "microflares" and "nanoflares" (Cargill \& Klimchuk 2004; Klimchuk 2006) is a popular hypothesis to explain the heating of the corona of the Sun and that of other stars. Their nanoflare model assumes that any coronal loop is composed of many unresolved strands of magnetic flux and that these strands are "heated impulsively by a small burst of energy (which, for convenience, are called a nanoflare, although the range of energies is completely arbitrary". Further, the strands are thermally isolated from each other and can be viewed as elemental magnetic flux tubes with a small value for the plasma beta (Cargill \& Klimchuk 2004). An individual heating event on such a strand leads to a dynamic evolution of the affected plasma located on this particular strand. In a typical observation of a star and even in a typical observation of the solar corona, a multitude of heating events occur more or less simultaneously on different strands located in the field of view or are averaged during a typical exposure time. Therefore, in this picture, any solar or stellar coronal observation represents a mean of the heating and cooling history of possibly a very large number of individual energy releases. For a computation of the global properties of solar and stellar coronae in terms of the distributions of plasma densities, temperatures, and flow velocities, a knowledge and modeling of the relevant heating and cooling processes is mandatory. Such models specifically assume an impulsive heating of the strands of magnetic flux, which can be recognized only in data with very high time resolution $(\sim 1 \mathrm{~s})$, and then cool, first by conduction and then by radiation (Cargill \& Klimchuk 2004). This is precisely what appears to have happened on CN Leo: An energy release on a time scale of less than $10 \mathrm{~s}$ in a single loop has to our knowledge never been observed in any star nor on the Sun. Therefore, with our high time-resolution, soft X-ray, and optical observations of the impulsive flare on CN Leo - by its energetics definitely not a nanoflare - we may have isolated the relevant physics for nanoflare heating of the corona of the Sun and the coronae of the stars.

Acknowledgements. This work is based on observations obtained with $X M M$-Newton, an ESA science mission with instruments and contributions directly funded by ESA Member States and the USA (NASA), and on observations collected at ESO, Paranal, Chile, under the program 076.D-0024(A). C.L., U.W., and B.F. acknowledge financial support by the DLR under 50OR0105, A.R. through an Emmy Noether Fellowship from DFG under DFG RE 1664/4-1, and F.R. and G.P. acknowledge support from Agenzia Spaziale Italiana and Italian Ministero dell'Università e della Ricerca. We finally thank M. Miceli for useful suggestions.

\section{References}

Betta, B., Peres, G., Reale, F., \& Serio, S. 1997, A\&AS, 122, 585 Brown, J. C. 1973, Sol. Phys., 28, 151

Cargill, P. J., \& Klimchuk, J. A. 2004, ApJ, 605, 911

Fisher, G. H. 1987, ApJ, 317, 502

Fuhrmeister, B., Schmitt, J. H. M. M., \& Wichmann, R. 2004 A\&A, 417, 701

Fuhrmeister, B., Liefke, C., \& Schmitt, J. H. M. M. 2007, A\&A, 468, 221

Holman, G. 2002, ApJ, 586, 606

Hudson, H. S., Strong, K. T., Dennis, B. R., et al. 1994, ApJ, 422, L25

Kahler, S. 1975, in Solar gamma-, X-, and EUV radiation, Proc. Symp., Buenos Aires, Argentina, June 11-14, 1974 (A76-10126 01-92) (Dordrecht: D. Reidel Publishing Co.), 211

Kostiuk, N. D., \& Pikelner, S. B. 1974, Astron. Zh., 51, 1002

Klimchuk, J. 2006, Sol. Phys., 234, 41

Kürster, M., \& Schmitt, J. H. M. M. 1996, A\&A, 311, 211

Neupert, W. M. 1968, ApJ, 153, L59

Osten, R. A., Drake, S., Tueller, J., et al. 2007, ApJ, 654, 1022

Parker, E. N. 1988, ApJ, 330, 474

Peres, G. 2000, Sol. Phys., 193, 33

Peres, G., Rosner, R., Serio, S., \& Vaiana, G. S. 1982, ApJ, 252, 791

Priest, E. R,. \& Forbes, T. G. 2002, A\&AR, 10, 313

Reiners, A., Schmitt, J. H. M. M., \& Liefke C. 2007, A\&A, 466, L13

Schmitt, J. H. M. M., Haisch, B. M., \& Barwig, H. 1993, ApJ, 419, L81

Somov, B. V., Syratovskii, S. I., \& Spektor, A. R. 1981, Sol. Phys., 73, 145

Somov, B. V., Kosugi, T., Litvinenko, Y. E., et al. 1997, ApJ, 485, 895

Syratovskii, S. I., \& Shmeleva, O. P. 1972, Sov. Astron., 273

Tamres, D. H., Canfield, R. C., \& McClymont, A. N. 1986, ApJ, 309, 409

Vernazza, J. E., Avrett, E. H., \& Loeser, R. 1981, ApJS, 45, 635

Vilmer, N., Trottet, G., Barat, C., et al. 1994, Space Sci. Rev., 68, 233 